Bull. Korean Math. Soc. 48 (2011), No. 3, pp. 611-616

DOI 10.4134/BKMS.2011.48.3.611

\title{
MINIMAL NONCOMMUTATIVE REVERSIBLE AND REFLEXIVE RINGS
}

\author{
Byung-OK Kim And YAng LeE
}

\begin{abstract}
The reflexiveness and reversibility were introduced by Mason and Cohn respectively. The structures of minimal reversible rings and minimal reflexive rings are completely determined. The term minimal means having smallest cardinality.
\end{abstract}

Throughout this note all rings are associative with identity unless otherwise stated. Let $R$ be a ring. The $n$ by $n$ full (resp. upper triangular) matrix ring over $R$ is denoted by $\operatorname{Mat}_{n}(R)$ (resp. $U_{n}(R)$ ). Let $J(R)$ denote the Jacobson radical of $R . \mathbb{Z}_{n}$ denotes the ring of integers modulo $n$.

According to Cohn [2], a ring $R$ is called reversible if $a b=0$ implies $b a=0$ for $a, b \in R$. Anderson-Camillo [1] used the term $Z C_{2}$ for the reversibility. A ring is called reduced if it has no nonzero nilpotent elements. Reduced rings are reversible via a simple computation. Commutative rings are clearly reversible, but the converse need not hold since there exist many kinds of noncommutative reduced rings. Note also that there are many kinds of non-reduced commutative rings (e.g., $\mathbb{Z}_{k^{n}}$ for any $k, n \geq 2$ ). A ring is called Abelian if every idempotent is central. Reversible rings are Abelian through a simple computation.

Due to Mason [12], a ring $R$ is called reflexive if $a R b=0$ implies $b R a=0$ for $a, b \in R$. Semiprime rings are reflexive by a simple computation. Reversible rings are clearly reflexive, but the converse need not hold since there exist many kinds of non-Abelian semiprime rings (e.g., $\operatorname{Mat}_{n}(R)$ for a semiprime ring $R$ and $n \geq 2$ ). Note that reflexive rings need not be Abelian as can be seen by $\operatorname{Mat}_{n}(R)(n \geq 2)$ over a semiprime ring $R$.

A ring $R$ is called semilocal if $R / J(R)$ is semisimple Artinian, and $R$ is called semiperfect if $R$ is semilocal and idempotents can be lifted modulo $J(R)$. R is called local if $R / J(R)$ is a division ring. Local rings are clearly semilocal, and one important case of semiperfect rings is when the Jacobson radical is nil

Received October 19, 2009.

2010 Mathematics Subject Classification. 16P10, $16 \mathrm{U} 80$.

Key words and phrases. minimal noncommutative reversible ring, minimal noncommutative reflexive ring.

This study was supported by the Research Fund Program of Research Institute for Basic Sciences, Pusan National University, Korea, 2010, Project No. RIBS-PNU-2010-104.

(C)2011 The Korean Mathematical Society 
by [10, Proposition 3.6.1]. Local rings are Abelian through a simple computation. Note that $J(R)$ is nilpotent by [9, Theorem 2.4.12] when $R$ is one-sided Artinian.

Lemma 1. (1) The class of reversible rings is closed under subrings (possibly without identity).

(2) Let $R_{i}(i \in I)$ be rings. Then the following conditions are equivalent:

(a) $R_{i}$ is reversible for all $i \in I$;

(b) The direct product of $R_{i}(i \in I)$ is reversible;

(c) The direct sum (possibly without identity) of $R_{i}(i \in I)$ is reversible.

(3) A ring $R$ is semiperfect and reversible if and only if $R$ is a finite direct sum of local reversible rings.

Proof. (1) It is obvious that the reversibility is hereditary to subrings.

(2) Let $R$ be the direct product of reversible rings $R_{i}(i \in I)$. Suppose that $\left(a_{i}\right)\left(b_{i}\right)=0$ for $\left(a_{i}\right),\left(b_{i}\right) \in R$. Then $a_{i} b_{i}=0$ for each $i \in I$. Since $R_{i}$ is reversible, $b_{i} a_{i}=0$ for each $i \in I$, entailing $\left(b_{i}\right)\left(a_{i}\right)=0$. The proofs of remainder come from the result (1).

(3) We apply the proof of [5, Lemma 2.2(3)]. Suppose that $R$ is a semiperfect reversible ring. Since $R$ is semiperfect, $R$ has a finite orthogonal set of local idempotents whose sum is 1 by [10, Proposition 3.7.2], say $R=\oplus \sum_{i=1}^{n} e_{i} R$ such that each $e_{i} R e_{i}$ is a local ring. Since reversible rings are Abelian, every $e_{i} R$ is an ideal of $R$ with $e_{i} R=e_{i} R e_{i}$. Each $e_{i} R$ is a reversible ring by the result (2). Conversely assume that $R$ is a finite direct sum of local reversible rings. Then $R$ is semiperfect since local rings are semiperfect, and moreover $R$ is reversible by the result (2).

We use $G F\left(p^{n}\right)$ to denote the Galois field of order $p^{n}$. Due to Feller [4], a ring is called right (left) duo if every right (left) ideal is two-sided. Right or left duo rings are clearly Abelian via a simple computation. Xue [13] proved that finite rings are right duo if and only if they are left duo. We first study minimal noncommutative reversible rings, analyzing the following example.

Example 2. Due to Xue [14, Example 2], let $R=\left\{\left(\begin{array}{cc}a & b \\ 0 & a^{2}\end{array}\right) \mid a, b \in G F\left(2^{2}\right)\right\}$ be a subring of $U_{2}\left(G F\left(2^{2}\right)\right)$. Then

$$
J(R)=\left\{\left(\begin{array}{ll}
0 & b \\
0 & 0
\end{array}\right) \mid b \in G F\left(2^{2}\right)\right\}, R / J(R) \cong G F\left(2^{2}\right),
$$

and $R$ is reversible by the computation in [5, Example 2.5]. In fact, letting $x y=0$ for $x, y \in R \backslash 0$, both $x$ and $y$ must be contained in $J(R)$; hence we also get $y x=0$. Thus $R$ is a reversible ring of order 16 . It is not difficult to check that $R$ is duo.

Theorem 3. If $R$ is a minimal noncommutative reversible ring, then $R$ is of order 16 and is isomorphic to the ring of all matrices of the form $\left(\begin{array}{cc}a & b \\ 0 & a^{2}\end{array}\right)$ over $G F\left(2^{2}\right)$. 
Proof. We apply the proof of [5, Theorem 2.6]. Eldridge proved, in [3, Proposition], that if a ring $A$ is of order $p^{3}, p$ a prime, then $A$ is isomorphic to $U_{2}(G F(p))$, and that if a finite ring has a cube free factorization, then it is commutative, in [3, Theorem]. Thus a minimal noncommutative reversible ring is of order 16, considering Example 2, since reversible rings are Abelian and $U_{2}(G F(p))$ is non-Abelian. Note that finite rings are semiperfect.

Let $R$ be a minimal noncommutative reversible ring. Then since $R$ is minimal, $R$ is a local ring with the help of Lemma 1(3). We have three cases of $|J(R)|=2,|J(R)|=4$ or $|J(R)|=8$, where $|J(R)|$ means the cardinality of $J(R)$. Note that $R / J(R)$ is a field and $J(R)$ is a vector space over $R / J(R)$. If $|J(R)|=2$ then $R / J(R) \cong G F\left(2^{3}\right)$ and so $|J(R)| \geq 8$, a contradiction. Suppose $|J(R)|=8$. Then $R / J(R) \cong G F(2)$ and by [7, Theorem 2.3.6], $J(R)$ has a basis $\{a, b, c\}$ over $G F(2)$ with $a^{2}=a b=c$ and $b a=0$; hence $R$ is not reversible, a contradiction.

Consequently we have the only case of $|J(R)|=4$, for $R$ to be a minimal noncommutative reversible ring. In this case $R$ is right duo (hence duo) by the argument in the proof of [5, Theorem 2.6]. This implies that $R$ is a noncommutative duo ring of order 16 with $|J(R)|=4$. Then $R$ is isomorphic to the ring in Example 2 by [14, Theorem 3].

Local rings need not be reversible by the first and second rings in [14, Example 2], and reversible rings also need not be local since there exist many semiprimitive reduced rings but not division rings (e.g., the ring of integers, the first Weyl algebra over a field of characteristic zero).

Lemma 4. (1) The class of reflexive rings is not closed under subrings.

(2) Let $R_{i}(i \in I)$ be rings. Then the following conditions are equivalent:

(a) $R_{i}$ is reflexive for each $i \in I$;

(b) The direct product of $R_{i}(i \in I)$ is reflexive;

(c) The direct sum (possibly without identity) of $R_{i}(i \in I)$ is reflexive.

(3) Let $R$ be an Abelian ring. Then $R$ is a semiperfect reflexive ring if and only if $R$ is a finite direct sum of local reflexive rings.

Proof. (1) By [8, Example 2.8].

(2) Let $R$ be the direct product of reflexive rings $R_{i}(i \in I)$. Suppose that $\left(a_{i}\right) R\left(b_{i}\right)=0$ for $\left(a_{i}\right),\left(b_{i}\right) \in R$. Then $a_{i} R_{i} b_{i}=0$ for each $i \in I$. Since $R_{i}$ is reflexive, $b_{i} R_{i} a_{i}=0$ for each $i \in I$, entailing $\left(b_{i}\right) R\left(a_{i}\right)=0$. Let $e=\left(e_{i}\right) \in R$ such that $e_{i}=1$ and $e_{j}=0$ for all $j \neq i$. Then $e^{2}=e$ and $e R e \cong R_{i}$. If $R$ is reflexive, then $R_{i}$ is also reflexive by [8, Theorem 2.7(1)]. The proof for the direct sum case is similar.

(3) The necessity is obtained by the same method as Lemma 1(3). Conversely assume that $R$ is a finite direct sum of local reflexive rings. Then $R$ is semiperfect since local rings are semiperfect, and moreover $R$ is reflexive by the result (2). 
Note that $\left\{\left(\begin{array}{cc}a & b \\ 0 & a^{2}\end{array}\right) \mid a, b \in G F\left(2^{2}\right)\right\}$ is reversible (hence reflexive) by Example 2, and that $\operatorname{Mat}_{2}\left(\mathbb{Z}_{2}\right)$ is reflexive since it is semiprime. || denotes the cardinality.

Theorem 5. If $R$ is a minimal noncommutative reflexive ring, then $R$ is a ring of order 16 such that $R$ is isomorphic to the ring of all matrices of the form $\left(\begin{array}{cc}a & b \\ 0 & a^{2}\end{array}\right)$ over $G F\left(2^{2}\right)$ when $R$ is Abelian, and $\operatorname{Mat}_{2}\left(\mathbb{Z}_{2}\right)$ when $R$ is non-Abelian.

Proof. For the proof of the case of Abelian, we apply one of [5, Theorem 2.6]. Eldridge proved that if a finite ring has a cube free factorization, then it is commutative, in $\left[3\right.$, Theorem]; and that if a $\operatorname{ring} A$ is of order $p^{3}, p$ a prime, then $A$ is isomorphic to $U_{2}(G F(p))$, in [3, Proposition]. Thus every minimal noncommutative ring is isomorphic to $U_{2}\left(\mathbb{Z}_{2}\right)$. But $U_{2}\left(\mathbb{Z}_{2}\right)$ is not reflexive by [8, Example 2.8], and so a minimal noncommutative reflexive ring is of order 16 , considering the reflexive rings $\left\{\left(\begin{array}{cc}a & b \\ 0 & a^{2}\end{array}\right) \mid a, b \in G F\left(2^{2}\right)\right\}$ and $\operatorname{Mat}_{2}\left(\mathbb{Z}_{2}\right)$.

First let $R$ be a minimal Abelian noncommutative reflexive ring. Then $R$ is a local ring by Lemma 4(3) since $R$ is minimal. Here we have three cases of $|J(R)|=2,|J(R)|=4$ or $|J(R)|=8$, where $|J(R)|$ means the cardinality of $J(R)$. Note that $R / J(R)$ is a field and $J(R)$ is a vector space over $R / J(R)$. The case of $|J(R)|=2$ is impossible by the proof of [5, Theorem 2.6]. Suppose $|J(R)|=8$. Then $R / J(R) \cong G F(2)$ and by [7, Theorem 2.3.6], $J(R)$ has a basis $\{a, b, c\}$ over $G F(2)$ with $a^{2}=a b=c$ and $b a=0$. Then since every sum-factor of each element in $b R a$ contains $b a$ in its product expression, $b R a$ must be zero. But $0 \neq a b \in a R b$ and so $R$ is not reflexive, a contradiction. Consequently we have the only case of $|J(R)|=4$, for $R$ to be a minimal Abelian noncommutative reflexive ring. In this case $R$ is isomorphic to $\left\{\left(\begin{array}{cc}a & b \\ 0 & a^{2}\end{array}\right) \mid a, b \in G F\left(2^{2}\right)\right\}$ by the proof of [5, Theorem 2.6].

Secondly let $R$ be a minimal non-Abelian (hence noncommutative) reflexive ring. Then $R$ is not local since local rings are Abelian.

By the Wedderburn-Artin theorem, $R / J(R)$ is isomorphic to $\sum_{i=1}^{n} \operatorname{Mat}_{k_{i}}\left(D_{i}\right)$ for some $k_{i}$ 's and finite fields $D_{i}$ 's. In fact, $D_{i}=\mathbb{Z}_{2}$ for all $i$ since $R$ is minimal. Assume that $k_{i}=1$ for all $i$. Here we have there cases of $|J(R)|=2,|J(R)|=4$, and $|J(R)|=8$.

If $|J(R)|=8$, then $R / J(R) \cong \mathbb{Z}_{2}$ and so $R$ is local, a contradiction.

Let $|J(R)|=2$. Then $R / J(R) \cong \mathbb{Z}_{2} \oplus \mathbb{Z}_{2} \oplus \mathbb{Z}_{2}$, and since $J(R)$ is nilpotent and $|J(R)|=2$ we have $J(R)^{2}=0$. We can obtain orthogonal nonzero idempotents $e_{1}, e_{2}, e_{3}$ with $e_{1}+e_{2}+e_{3}=1$ by [10, Proposition 3.7.2], and we have

$$
R=\{x+y \mid x \in I, y \in J(R)\}
$$

where $I=\left\{0,1, e_{1}, e_{2}, e_{3}, 1-e_{1}, 1-e_{2}, 1-e_{3}\right\}$. Note that idempotents commute each other. Since $R$ is non-Abelian, there exists $e \in\left\{e_{1}, e_{2}, e_{3}, 1-e_{1}, 1-e_{2}, 1-\right.$ $\left.e_{3}\right\}$ and $r \in R$ such that $e r-r e \neq 0$ (otherwise, $R$ is commutative). Consider the subset $e(e r-r e) R e$. Note that $e r-r e \in J(R), e(e r-r e) e=0$, and every element in $e(e r-r e) R e$ is of the form $e(e r-r e)(f+j) e$, where $f \in I$ and 
$j \in J(R)$. But since $E=\left\{e_{1}, e_{2}, e_{3}\right\}$ is orthogonal and $J(R)^{2}=0$, we get

$$
e(e r-r e)(f+j) e=e(e r-r e) j e=0 \text { when } f \in E \text { with } f \neq e
$$

and

$e(e r-r e)(f+j) e=e(e r-r e) e e+e(e r-r e) j e=e(e r-r e) e=0$ when $f=e$.

These entail $e(e r-r e) R e=0$. Now since $R$ is reflexive, $e R e(e r-r e)=0$ and this implies $e r-e r e=0$. Next consider the subset $e R(e r-r e) e$. Every element in $e R(e r-r e) e$ is of the form $e(f+j)(e r-r e) e$, where $f \in I$ and $j \in J(R)$. Similarly $e(f+j)(e r-r e) e=0$ and this yields $e R(e r-r e) e=0$. Also since $R$ is reflexive, $(e r-r e) e R e=0$ and this implies $r e-e r e=0$. Consequently we get $e r=e r e=r e$, a contradiction.

Let $|J(R)|=4$. Then $R / J(R) \cong \mathbb{Z}_{2} \oplus \mathbb{Z}_{2}$. Since $|J(R)|=4$ and $J(R)$ is nilpotent, we have one of the following three cases with the help of [7, Theorems 2.3.2 and 2.3.3] and their proofs:

(1) There exists a basis $\{a, b\}$ for an additive abelian group $J(R)$ such that char $a=\operatorname{char} b=2$ and $a^{2}=b^{2}=a b=b a=0$ (equivalently, $J(R)^{2}=0$ );

(2) There exists a basis $\{a, b\}$ for an additive abelian group $J(R)$ such that $a^{2}=b, a^{3}=0$ and $J(R)=\langle a\rangle$, where $\langle a\rangle$ is the subring of $J(R)$ generated by $a$;

(3) There exists an element $a \in J(R)$ such that $J(R)=\{0, a, 2 a, 3 a\}$ and $\operatorname{char} a=4$.

In this case of $|J(R)|=4$, we also obtain nonzero idempotents $e_{1}$ and $e_{2}$, such that $e_{1}+J(R)=(1,0)+J(R)$ and $e_{2}+J(R)=(0,1)+J(R)$, with the help of [10, Proposition 3.6.2]. Note $e_{1} e_{2}, e_{2} e_{1} \in J(R)$. Further, we have

$$
R=\{x+y \mid x \in J, y \in J(R)\}
$$

where $J=\left\{0,1, e_{1}, e_{2}\right\}$. Since $R$ is non-Abelian, there exists $e \in\left\{e_{1}, e_{2}\right\}$ and $r \in R$ such that $e r-r e \neq 0$ (if $e_{1}, e_{2}$ are both central, then we can find another such set $\left\{e_{1}, e_{2}\right\}$ in which $e_{1}$ or $e_{2}$ is non-central). Note that $e r-r e \in J(R), e(e r-r e) e=0$, and every element in $e(e r-r e) R e$ is of the form $e(e r-r e)(f+j) e$, where $f \in J$ and $j \in J(R)$.

Consider the subset $e J(R) e$. Then we have three cases of $|e J(R) e|=0$, $|e J(R) e|=2$ or $|e J(R) e|=4$ (i.e., $e J(R) e=J(R))$.

If $|e J(R) e|=0$, then $e(e r-r e) R e=0$ from $e r-r e \in J(R)$.

If $|e J(R) e|=4$ (i.e., $e J(R) e=J(R))$, then $e r-r e=e(e r-r e) e=0$ from $e r-r e \in J(R)$ and so $e(e r-r e) R e=0$.

Let $|e J(R) e|=2$. Then $(e J(R) e)^{2}=0$ clearly. Consider the cases of $(2)$ and (3). Then the elements in $J(R)$ commute and so for every $e(e r-r e)(f+j) e \in$ $e(e r-r e) R e$, we get

$$
\begin{aligned}
e(e r-r e)(f+j) e & =e(e r-r e) f e+e(e r-r e) j e \\
& =e(e r-r e) f e+e j e(e r-r e) e \\
& =e(e r-r e) f e=e f e(e r-r e) e=0
\end{aligned}
$$


since $(e r-r e) \in J(R)$ (for, $e(e r-r e) f e=0$ when $f \in\{0,1, e\}$, and $f e \in J(R)$ when $f \in\left\{e_{1}, e_{2}\right\}$ and $f \neq e$.) This yields $e(e r-r e) R e=0$. Consider the case of (1). Then we have $J(R)^{2}=0$ and so $e(e r-r e)(f+j) e=e(e r-r e) f e=0$ by the same reason as before, entailing $e(e r-r e) R e=0$.

Summarizing, we get $e(e r-r e) R e=0$ in any case of (1), (2) and (3). Since $R$ is reflexive, $e \operatorname{Re}(e r-r e)=0$ and so we have $e r=e r e$. These yield $r e=e r e=e r$, a contradiction to $e r-r e \neq 0$.

Therefore $k_{i} \geq 2$ for some $i$, say $i=1$. But $R$ is of order 16 and so we must have $R / J(R) \cong \operatorname{Mat}_{2}\left(\mathbb{Z}_{2}\right)$, entailing $J(R)=0$. This concludes $R \cong$ $\operatorname{Mat}_{2}\left(\mathbb{Z}_{2}\right)$.

\section{References}

[1] D. D. Anderson and V. Camillo, Semigroups and rings whose zero products commute, Comm. Algebra 27 (1999), no. 6, 2847-2852.

[2] P. M. Cohn, Reversible rings, Bull. London Math. Soc. 31 (1999), no. 6, 641-648.

[3] K. E. Eldridge, Orders for finite noncommutative rings with unity, Amer. Math. Monthly 73 (1966), 376-377.

[4] E. H. Feller, Properties of primary noncommutative rings, Trans. Amer. Math. Soc. 89 (1958), 79-91.

5] C. Huh, H. K. Kim, N. K. Kim, and Y. Lee, Basic examples and extensions of symmetric rings, J. Pure Appl. Algebra 202 (2005), no. 1-3, 154-167.

[6] N. K. Kim and Y. Lee, Extensions of reversible rings, J. Pure Appl. Algebra 185 (2003), no. 1-3, 207-223.

[7] R. Kruse and D. Price, Nilpotent Rings, Gordon and Breach, New York, 1969.

[8] T. K. Kwak and Y. Lee, Reflexive property of rings, submitted.

[9] T. Y. Lam, Lectures on Modules and Rings, Springer-Verlag, New York, 1999.

[10] J. Lambek, Lectures on Rings and Modules, Blaisdell Publishing Company, Waltham, 1966.

[11] G. Marks, Reversible and symmetric rings, J. Pure Appl. Algebra 174 (2002), no. 3, 311-318.

[12] G. Mason, Reflexive ideals, Comm. Algebra 9 (1981), no. 17, 1709-1724.

[13] W. Xue, On strongly right bounded finite rings, Bull. Austral. Math. Soc. 44 (1991), no. $3,353-355$.

[14] _ Structure of minimal noncommutative duo rings and minimal strongly bounded nonduo rings, Comm. Algebra 20 (1992), no. 9, 2777-2788.

BYUNG-OK KIM

Department of Mathematics

Korea SCIEnCE ACADEMy

PusAn 614-103, Korea

E-mail address: bokim26@hanmail.net

YANG LEE

Department of Mathematics

Pusan National University

Pusan 609-735, KoreA

E-mail address: ylee@pusan.ac.kr 